\title{
COMPARISON OF THREE DEVICES FOR MEASURING THE SECOND DERIVATIVE OF A LANGMUIR PROBE CURVE
}

\author{
by S. C. M. LUIJENDIJK and J. VAN ECK \\ Fysisch Laboratorium der Rijksuniversiteit, Utrecht, Nederland
}

\section{Synopsis}

Wo have compared three devices, employing different principles, which plot the second derivative (SD) of the current voltage curve of a Langmuir probe. The probe measurements were performed in the positive column of a helium discharge. Two devices, using low modulation frequencies, produced within $1 \%$ the same shape of the SD curve. The third $(200 \mathrm{kHz})$ yielded 10 to $25 \%$ lower values in the maximum of the SD curve; the discrepancy depended upon the pressure and diminished towards more negative probe potentials. The influence on the SD curves of the magnitude of the modulation voltages (up to $1 \mathrm{~V}$ peak to peak) is negligible in the case of helium. The determination of the space potential using the SD curves is discussed.

I. Introduction. The electron velocity distribution function is an important characteristic of a discharge and a great deal of experimental work has been devoted to the determination of this function. Druyvesteyn's method ${ }^{1}$ ), obtaining the energy distribution of electrons in ionized gases from the second derivate (SD) of the current-voltage curve of the probe is now fully appreciated and applied by several authors ${ }^{2-6}$ ). A device for taking the SD impresses, for instance, a small sinusoidal voltage of a certain frequency on the steady voltage which is the probe bias. The resulting probe current obtains a periodic component, which is not purely sinusoidal because the probe current is not linear on the voltage. The amplitude of the second harmonic hereof is a measure for the deformation, which is proportional to the required SD of the probe curve. The probe bias is varied very slowly with a sawtooth generator in order to measure the SD as a function of the voltage.

Several investigators use automatic SD devices working on different principles, which in some cases lead to differences in the results. In the course of designing a suitable apparatus for our work we developed three different devices:

$1^{\circ}$ Second harmonic method. A small sinusoidal signal is superimposed on the probe with a frequency of $83.3 \mathrm{~Hz}$. Detection: $166.7 \mathrm{~Hz}$.

$2^{\circ}$ Intermodulation method. Two small sinusoidal signals of 500 and 666.7 
$\mathrm{Hz}$ are superimposed on the probe together. The difference signal $-166.7 \mathrm{~Hz}$ - is detected.

$3^{\circ}$ High frequency modulation method. A $70 \%$ modulated wave, with carrier frequency of $200 \mathrm{kHz}$ and modulation frequency of $1428.6 \mathrm{~Hz}$, is applied to the probe circuit. The $1428.6 \mathrm{~Hz}$ signal is detected.

In fig. 1 a picture is given of the periodic voltages used in the three devices. In the following we define the value of the term modulation voltage as the peak to peak voltage indicated in fig. 1. For us it was of interest to know if the frequency influenced the resulting SD curve, so we compared these three devices mutually. Furthermore the influence of the modulation voltage on the shape of the SD curves is measured.

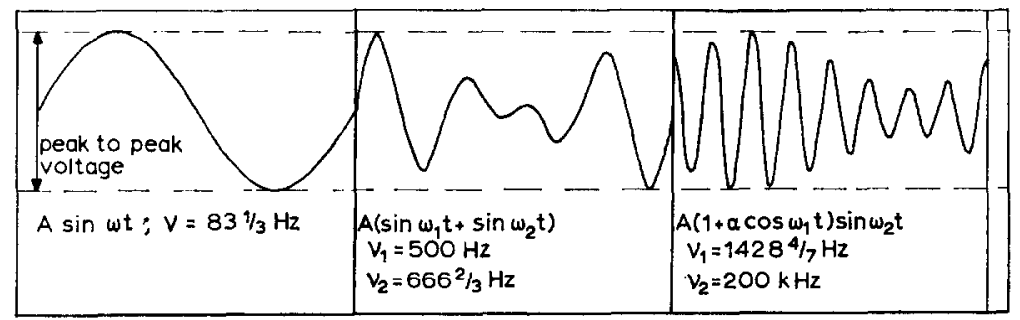

Fig. 1. The periodic voltages used in the different devices.

II. Device description. Device 1 was built following the specifications given by Branner, Friar and Medicus ${ }^{5}$ ) with a frequency of $1000 \mathrm{~Hz}$ and detection at $2000 \mathrm{~Hz}$. In the original setup it was very difficult to suppress the second harmonic already generated in the apparatus. Later on it turned out that higher harmonics of the $50 \mathrm{~Hz}$ ac supply interfered also with the detected frequency, so the $83.3 \mathrm{~Hz}$ frequency was chosen. Thus we got a better result which was improved by the use of another wiring diagram. Device 2 was developed, according to a personal suggestion of Medicus, with two frequencies of 500 and $666.7 \mathrm{~Hz}$. This combination was chosen because the detected frequency $(166.7 \mathrm{~Hz})$ is considerably lower than the two bcat frequencies, which has the advantage that these frequencies and their higher harmonics cannot interfere with the detected frequency. The third device was developed by means of the idea originally proposed by Malyshev ${ }^{4}$ ). In this case an all transistor apparatus was built. With all three devices it is possible to measure also the probe curve itself.

The frequencies used $(83.3,500,666.7$ and $1428.6 \mathrm{~Hz})$ were derived from a frequency standard $\left(10^{3}\right.$ and $\left.10^{4} \mathrm{~Hz}\right)$ by frequency devision. In devices 1 and 2 the difficulties with the $50 \mathrm{~Hz}$ of the ac supply were more serious than in device 3 because the measuring frequency $-166.7 \mathrm{~Hz}-$ and the third harmonic of the ac supply interfered with each other. 
We accurately measured the linearity between in- and output of the three devices. The comparison was performed with the detection frequency put on the probe line. We found deviations from linearity of $1 \%$ or less varying the input from zero tothe signals used normally. The linearity of the amplifiers was checked separately; deviations were found to be less than $1 \%$.

Device 1 and 2. In order to measure the SD (system 1) a small sinusoidal signal $(83.3 \mathrm{~Hz})$ was put over a resistor $R_{\mathbf{3}}$ in series with the probe, while $R_{\mathbf{2}}$ was short circuited (see fig. 2). In system 2 two beat frequencies (500 and $666.7 \mathrm{~Hz}$ ) were put over $R_{\mathbf{3}}$ and $R_{\mathbf{2}}$. In both cases we detected the same frequency $(166.7 \mathrm{~Hz})$ over $R_{1}$, so that we could use the same detection unit. The detected signal passes a narrow band filter, in which the higher harmonics and the starting frequencies $(83.3,500$ and $666.7 \mathrm{~Hz})$ are reduced by at least $20 \mathrm{~dB}$, dependent on the used $R_{\mathbf{1}}$. Next the signal was amplified and phase sensitive detected by a synchronous detector. The band width of the 166.7 $\mathrm{Hz}$ amplifier was about $4 \mathrm{~Hz}$. The resulting voltage was rectified and measured on an $\mathrm{X}-\mathrm{Y}$ recorder (Mosely $7030 \mathrm{AR}$ input impedance $1 \mathrm{M} \Omega$ ). The maximum gain of the amplifiers was of the order of 25000 . The probe current itself could be measured directly over a variable resistance of 1 $500 \Omega$. We normally used a resistance of 5 or $10 \Omega$.

Device 3. The third device consists of a $70 \%$ modulated wave with carrier frequency of $200 \mathrm{kHz}$ from an internal oscillator and modulation frequency of $1428.6 \mathrm{~Hz}$. A block diagram of the apparatus is shown in fig. 3.

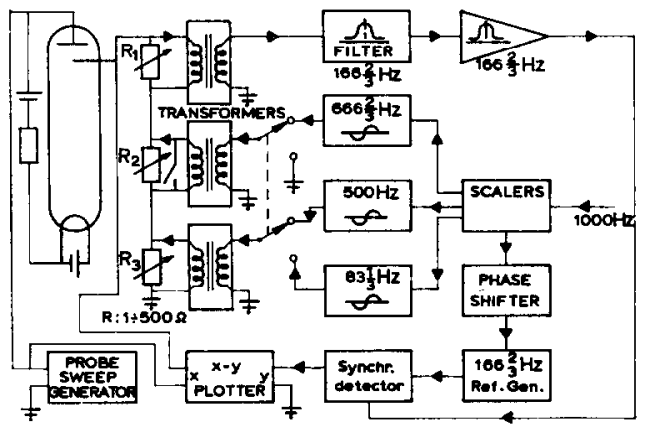

Fig. 2. Block diagram of the SD devices 1 and 2.

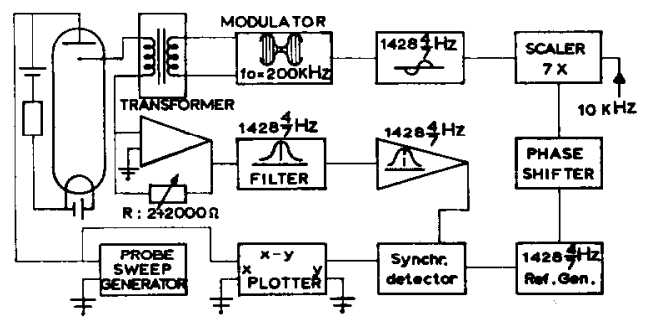

Fig. 3. Block diagram of device 3 . 
The important difference with the other two devices is that the impedance of the external part of the probe circuit is in the order of $0.1 \Omega$ or less (also for the $200 \mathrm{kHz}$ ) because an operational amplifier was used (amplification $5000 \times$ ) over the measuring resistance $(2-2000 \Omega)$; so the voltage drop over the measuring resistance was compensated. Normally a measuring resistance of $10 \Omega$ was used. The frequency of the modulation signal $(1428.6 \mathrm{~Hz})$ was chosen well above the $50 \mathrm{~Hz}$ frequency of the ac supply. The $70 \%$ modulated $200 \mathrm{kHz}$ voltage was put on the probe via an anti-1428.6 Hz filter and a hig! frequency transformer. The amplitude of the $1428.6 \mathrm{~Hz}$ component in the probe current was measured over $R$ via a band pass filter and two tuned arnplifiers $(250.000 \times)$ in a phase sensitive synchronous detector.

III. Apparatus. The discharge tube was a $4 \mathrm{~cm}$ diameter glass tube with a length of about $85 \mathrm{~cm}$. The measurements were performed in the positive column of a helium discharge with a pressure of about 0.3 torr; tube currents of 100-400 $\mathrm{mA}$ were employed. The discharge tube was thoroughly outgassed and baked at $450^{\circ} \mathrm{C}$ for over 24 hours; we reached a rest gas pressure of about $5 \times 10^{-9}$ torr. The $\mathrm{Ba}$ oxide coated filament was thoroughly outgassed in the course of the formation; the anode was a molybdenum plate. The helium was purified by leaking it through a heated quartz tube. The measurements were made with two cylindrical probes (length 3.5 and $15.0 \mathrm{~mm}$, diameter $0.052 \mathrm{~mm}$ ). The probes were placed $5 \mathrm{~cm}$ from one another at the axis of the tube, about $15 \mathrm{~cm}$ from the anode. The measurements were carried out with the anode as a reference. We mainly used the long probe; however, measurements with the shorter probe yielded identical results concerning the comparison of the devices.

IV. Measurements and results. The measuring procedure was that data were taken with the three devices each after the other; in the meanwhile the conditions of the discharge tube were kept constant very precisely. The SD curve was made with an $\mathrm{X}-\mathrm{Y}$ recorder (see fig. 2 and 3 ); the probe bias voltage was supplied by a slow sawtooth generator ( $40 \mathrm{~V}$ in $45 \mathrm{~s}$ ).

IVa. Influence of the modulation voltage. We compared the SD curves, with the long cylinder probe, varying the modulation voltage from $0.4 \mathrm{~V}$ to $1.0 \mathrm{~V}$ (peak to peak) with the $83.3 \mathrm{~Hz}$ device. The tube conditions were $p=0.23$ torr; tube current $100 \mathrm{~mA}$. The results given in table $\mathrm{I}$, are calculated from the measured SD curves which were normalized by making the sum of all the values of different rows equal to one another to get rid of the little differences in the amplifier gain. For our purpose only the relative shape of the curve is important as well as for the derivation of the electron velocity distribution from the SD curve. As is shown in table $I$ the differences between successive modulation voltages are negligible (errors of 0.2 are due to the evaluation of the plotted curves). So it is clear that in our 
TABLE I

\begin{tabular}{|c|c|c|c|c|}
\hline \multicolumn{5}{|c|}{$\begin{array}{l}\text { Comparison of the values of the SD curves measured } \\
\text { with the same probe under the same discharge con- } \\
\text { ditions, using different modulation voltages }(1,0.8 \text {, } \\
0.6 \text { and } 0.4 \mathrm{~V}) \text { with device } 1\end{array}$} \\
\hline$V$ (volt) & $1 \bar{V}$ & $0.8 \mathrm{~V}$ & $0.6 \mathrm{~V}$ & $0.4 \mathrm{~V}$ \\
\hline 0 & 0 & 0 & 0 & 0 \\
\hline 1 & 100.0 & 100.0 & 99.9 & 98.5 \\
\hline 2 & 93.2 & 92.9 & 93.1 & 93.0 \\
\hline 3 & 81.2 & 81.2 & 82.0 & 82.6 \\
\hline 4 & 70.3 & 69.8 & 69.9 & 70.8 \\
\hline 5 & 59.4 & 59.3 & 59.0 & 59.5 \\
\hline 6 & 50.0 & 50.0 & 49.8 & 50.4 \\
\hline 7 & 42.0 & 41.7 & 41.6 & 41.9 \\
\hline 8 & 34.8 & 34.5 & 34.7 & 34.7 \\
\hline 9 & 29.0 & 28.8 & 29.0 & 29.1 \\
\hline 10 & 24.3 & 24.1 & 24.0 & 24.1 \\
\hline 11 & 20.4 & 20.3 & 20.3 & 20.0 \\
\hline 12 & 17.1 & 17.1 & 16.9 & 17.1 \\
\hline 13 & 14.5 & 14.5 & 14.3 & 14.3 \\
\hline 14 & 12.4 & 12.4 & 12.4 & 12.2 \\
\hline 15 & 10.7 & 10.9 & 10.8 & 10.7 \\
\hline 16 & 9.0 & 9.1 & 9.1 & 9.0 \\
\hline 17 & 7.5 & 7.7 & 7.7 & 7.5 \\
\hline 18 & 6.2 & 6.3 & 6.3 & 6.2 \\
\hline 19 & 5.0 & 5.1 & 5.3 & 5.0 \\
\hline 20 & 4.1 & 4.2 & 4.2 & 4.1 \\
\hline 21 & 3.2 & 3.4 & 3.4 & 3.2 \\
\hline 22 & 2.3 & 2.7 & 2.7 & 2.3 \\
\hline 23 & 1.6 & 2.0 & 2.0 & 2.0 \\
\hline 24 & 1.3 & 1.4 & 1.4 & 1.4 \\
\hline 25 & 0.8 & 1.1 & 1.0 & 0.9 \\
\hline 26 & 0.6 & 0.7 & 0.7 & 0.7 \\
\hline 27 & 0.5 & 0.6 & 0.6 & 0.4 \\
\hline
\end{tabular}

case, where the electron temperature is of the order of $5 \mathrm{~V}$, a modulation voltage of $1 \mathrm{~V}$ produces the same results as lower values do. Call7) has calculated that for a ratio of the modulation voltage and electron temperature of 0.2 , the measured SD cannot bc distinguished from the true SD; this is confirmed by our measurements. In the following we report all our measurements with a modulation voltage of $0.8 \mathrm{~V}$.

We also measured the horizontal shift $\Delta V$ of the maximum of the SD curve, with respect to the maximum of the true SD curve (see fig. 4) measured as a function of increasing modulation voltage. $\mathrm{Call}^{7}$ ) has calculated this effect for an exponential function ending in a straight line. We made measurements with our $200 \mathrm{kHz}$ device, varying the modulation voltage from $2.35 \mathrm{~V}$ down to $0.4 \mathrm{~V}$. From the results, presented in table II, it follows that for the larger values the difference $\Delta V$ is just about half the modulated voltage which has also been calculated by Call for circumstances correspond- 


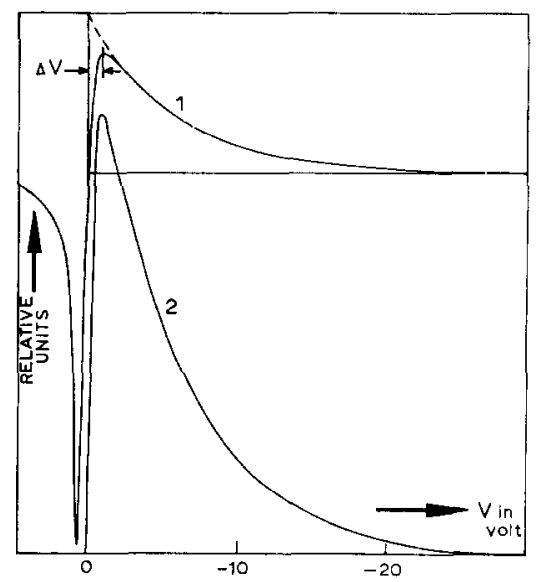

Fig. 4. A picture of a SD curve at both sides of the space potential is given together with an enlarged fraction for negative voltages. The dashed part is a hypothetical extrapolation of a SD curve for the limiting case of zero modulation voltage.

TABLE II

Comparison of the voltage difference $(\Delta V)$ between the maximum and the zero cross of the
$S D$ curve for different modulation voltages $V_{0}$
\begin{tabular}{|l|c|c|c|c|c|c|c|c|}
\hline $\begin{array}{l}\text { Co } \\
V_{0} \text { in volts } \\
\text { peak to peak }\end{array}$ & 0.4 & 0.5 & 0.8 & 1.0 & 1.6 & 2.0 & 2.35 & 2.5 \\
\hline$\Delta V$ in volts & $0.7 \pm 0.2$ & 0.8 & 0.8 & 0.8 & 1.0 & 1.0 & 1.1 & 1.2 \\
\hline
\end{tabular}

ing to ours. For the smaller modulation voltages the differences are somewhat too big, probably owing to our detection apparatus.

IV $b$. Space potential determination. The space potential is mostly found from the break in the semi logarithmic plot of the probe characteristic. Our SD device indicates unambiguously the bend in the probe characteristic, because the SD is zero here. We compared these two methods extensively and we found within 0.1 or $0.2 \mathrm{~V}$ the same potential for the zero cross of the $\mathrm{SD}$ curves and the break in the semi-logarithmic curve of the probe characteristic, which is illustrated in fig. 5 curve $B$ and $E$. As a consequence we take the space potential to be the potential where the SD curve crosses the zero line.

Furthermore, we have verified that the zero crossing is nearly independent of the modulation voltage. Measurements with modulation voltages of $0.2 \rightarrow 1 \mathrm{~V}$ showed a difference of about $0.1 \mathrm{~V}$ in the zero crossing.

IVc. Electron temperature measurement. A calculation of the electron temperature $T_{e}$ from the probe curve itself is only possible if we correct the measured total current $i_{p}$ by subtracting the ion current. In the case of helium the correction is necessary because the ions are relatively fast. 


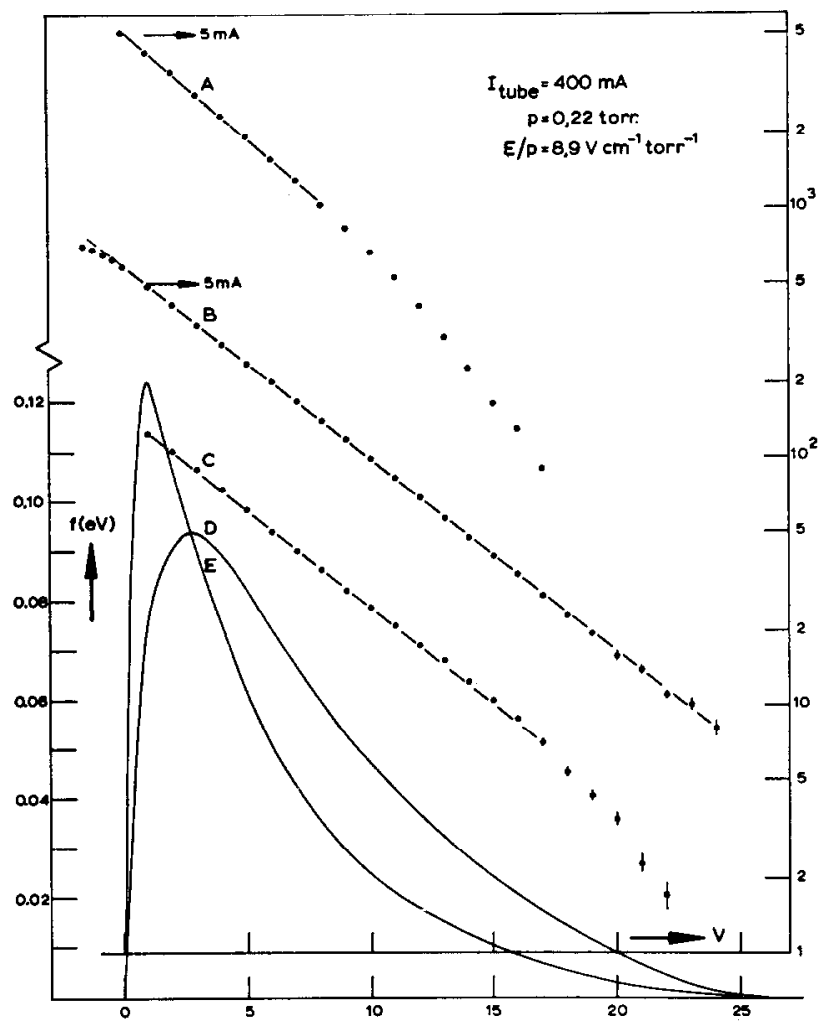

Fig. 5. Comparison of the semi-logarithmic plot of:

A: Probe current corrected by the form $C_{1} V^{0.6}$

Result: $k T_{e} / e=5.05 \mathrm{~V}$

B: Probe current corrected by a constant number

Result: $k T_{e} / e=5.58 \mathrm{~V}$

C: The SD belonging to the probe current.

Result: $k T_{e} / e=5.53 \mathrm{~V}$

together with:

D: The normalized electron energy distribution calculated from E.

$\mathrm{E}$ : The measured SD.

From the logarithm of the SD curve (fig. 5 curve C) measured with device 2 , which is a straight line over $17 \mathrm{~V}$ we conclude that the probe curve has the following shape:

$$
i_{p}=c_{0} \exp \left(-e V \mid k T_{e}\right)+c_{1} V+c_{2}
$$

in which $c_{0}, c_{1}$ and $c_{2}$ are constants.

Experimentally we found that $c_{1}$ is zero, because if we add only a constant number to the measured probe current we find already a straight line in a semi-logarithmic plot: see curve B fig. 5 in which $i_{p}-c_{2}$ is plotted against $V$. The data for this curve were taken from the first case in table III $(E / p=$ 
$=8.9 \mathrm{~V} \mathrm{~cm}^{1}$ torr $^{1}$ ) fourth row in which $c_{0}=5.08 \mathrm{~mA}$ and $c_{2}=-0.75$ $\mathrm{mA}$. One would expect a probe current corresponding to a Maxwell distribution of the electrons minus a correction that is proportional to the ion current. We are not able to say that in expression 1 the term with $c_{0}$ is concerned with the electrons and the term $c_{2}$ with the ions, for it is not plausible that the ion current should be independent of $V$. Now we suppose that the term with $c_{0}$ may be split up in an ion- and electron part, in such a way that at space potential the ion current becomes zero. The result is then:

Electron current

$$
i_{p e}=i_{0} \exp \left(\mathrm{eV} / k T_{e}\right)
$$

in which $i_{0}$ is the current at space potential.

Ion current

$$
i_{p i}=c_{2}\left(1-\exp \left(-\mathrm{eV} / k T_{e}\right)\right.
$$

with $i_{0}=c_{0}+c_{2}$.

It is reasonable to believe that the expression (3) is a function which very nearly equals the ion current. However, it is not clear yet why the

TABLE III

Comparison of the $S D$ curves $\left(\mathrm{d}^{2} i_{p} / \mathrm{d} V^{2}\right)$ for the three devices each at several different discharge conditions. The probe current corrected by a constant number is also given as well as the electron temperature in volts calculated from the slope of the $S D$ - and probe curve. $V=$ probe voltage (volts); $i_{p}=$ measured probe current $(\mathrm{mA}) ; 1,2,3=S D$ values measured by the different systems (arbitrary units)

\begin{tabular}{|c|c|c|c|c|c|c|c|c|c|c|c|c|}
\hline \multicolumn{5}{|c|}{$\begin{array}{c}p=0.22 \text { torr } I_{\text {tube }}=400 \mathrm{~mA} \\
E / p=8.9 \mathrm{~V} \mathrm{~cm}^{-1} \text { torr }^{-1}\end{array}$} & \multicolumn{4}{|c|}{$\begin{array}{c}p=0.35 \text { torr } I_{\text {tube }}=200 \mathrm{~mA} \\
E / p=6.7 \mathrm{~V} \mathrm{~cm}^{-1} \text { torr }^{-1}\end{array}$} & \multicolumn{4}{|c|}{$\begin{array}{c}p=0.35 \text { torr } I_{\text {tube }}=310 \mathrm{~mA} \\
E / P=6.7 \mathrm{~V} \mathrm{~cm}^{-1} \text { torr }^{-1}\end{array}$} \\
\hline $\mathrm{V}$ & 1 & 2 & 3 & $i_{p}+0.75$ & 1 & 2 & 3 & $i_{p}+0.575$ & 1 & 2 & 3 & $i_{p}+0.81$ \\
\hline 0 & 0 & 0 & 0 & 5.83 & 0 & 0 & 0 & 3.935 & 0 & 0 & 0 & 5.41 \\
\hline 1 & 124.0 & 124.0 & 110.0 & 4.89 & 123.5 & 124.0 & 104.7 & 3.205 & 133.0 & 135.4 & 109.5 & $4.4 \mathrm{l}$ \\
\hline 2 & 107.5 & 107.0 & 97.5 & 4.12 & 112.5 & 113.5 & 98.4 & 2.595 & 116.0 & 115.0 & 100.0 & 3.56 \\
\hline 3 & 89.5 & 90.0 & 83.5 & 3.42 & 91.8 & 90.7 & 85.2 & 2.100 & 92.5 & 94.0 & 85.5 & 2.86 \\
\hline 4 & 73.6 & 74.5 & 71.2 & 2.88 & 74.9 & 75.0 & 72.0 & 1.700 & 76.3 & 75.6 & 72.5 & 2.33 \\
\hline 5 & 60.8 & 61.5 & 60.5 & 2.41 & 61.2 & 61.0 & 60.0 & 1.375 & 61.5 & 61.8 & 60.9 & 1.88 \\
\hline 6 & 50.0 & 50.0 & 50.0 & 2.01 & 50.0 & 50.0 & 50.0 & 1.125 & 50.0 & 50.0 & 50.0 & 1.52 \\
\hline 7 & 41.9 & 42.0 & 42.3 & 1.69 & 41.1 & 40.8 & 41.4 & 0.920 & 40.6 & 40.4 & 41.1 & 1.235 \\
\hline 8 & 35.0 & 35.1 & 35.6 & 1.41 & 33.1 & 33.2 & 33.9 & 0.748 & 33.0 & 32.8 & 34.1 & 1.01 \\
\hline 9 & 29.6 & 29.1 & 30.2 & 1.19 & 27.0 & 27.0 & 28.2 & 0.600 & 26.8 & 26.8 & 27.8 & 0.81 \\
\hline 10 & 24.9 & 25.0 & 25.8 & 0.99 & 22.1 & 22.0 & 23.2 & 0.493 & 21.8 & 21.7 & 23.0 & 0.66 \\
\hline 11 & 21.2 & 21.0 & 22.1 & 0.83 & 17.9 & 17.9 & 19.4 & 0.400 & 17.8 & 17.8 & 18.9 & 0.54 \\
\hline 12 & 17.9 & 17.6 & 19.0 & 0.69 & 14.8 & 14.4 & 16.0 & 0.323 & 14.2 & 14.2 & 15.2 & 0.44 \\
\hline 13 & 15.0 & 14.9 & 16.1 & 0.57 & 11.8 & 11.8 & 12.9 & 0.260 & 11.8 & 11.6 & 12.5 & 0.36 \\
\hline 14 & 12.6 & 12.5 & 15.6 & 0.48 & 9.6 & 9.6 & 10.4 & 0.213 & 9.3 & 9.3 & 10.0 & 0.28 \\
\hline 15 & 10.6 & 10.5 & 11.7 & 0.40 & 7.6 & 7.5 & 8.3 & 0.175 & 7.4 & 7.4 & 7.8 & 0.22 \\
\hline 16 & 8.8 & 8.8 & 9.7 & 0.34 & 6.0 & 5.9 & 6.5 & 0.140 & 5.7 & 5.7 & 6.0 & 0.18 \\
\hline 17 & 6.9 & 7.0 & 7.9 & 0.28 & 4.6 & 4.4 & 4.9 & 0.113 & 4.2 & 4.5 & 4.5 & 0.15 \\
\hline 18 & 5.6 & 5.3 & 6.3 & 0.23 & 3.4 & 3.2 & 3.8 & 0.097 & 3.1 & 3.3 & 3.2 & 0.12 \\
\hline 19 & 4.4 & 4.3 & 5.0 & 0.20 & 2.6 & 2.4 & 2.7 & 0.077 & 2.2 & 2.3 & 2.2 & 0.10 \\
\hline 20 & 3.5 & 3.5 & 3.9 & 0.16 & 1.8 & 1.8 & 1.9 & 0.063 & 1.5 & 1.7 & 1.5 & 0.08 \\
\hline \multicolumn{2}{|c|}{$k T_{e} / e$} & 5.53 & & 5.58 & & 4.84 & & 4.79 & & 4.75 & & 4.77 \\
\hline
\end{tabular}

Discharge conditions 
electron temperature should have such a big influence on the ion current (this has to be explored). It seems justified, however, to conclude already that the electron energy distribution corresponds to the expression 2 for $i_{p e}$. Other measurements in this tube showed the same result (see table III).

Formely we used the following correction method:

We measured the current from $-200 \mathrm{~V}$ up to space potential $(0 \mathrm{~V}) . \mathrm{Up}$ to $-30 \mathrm{~V}$ the observed curve is mainly due to ions. We plotted this curve on log-log paper and found a straight line up to $-30 \mathrm{~V}$, which was extrapolated to $1 \mathrm{~V}$. The extrapolation was used for the correction of the probe current. We found for the corrected current (= "electron current") a more or less straight curve on a semi-log scale (curve A fig. 5). However, the slope of the "straight" part of this curve is at least 10\% smaller as compared to the slope of the SD curve (see curve A and C). This means that curve A has no true straight part.

We are now able to calculate the electron temperature from the slope of the curves $\mathrm{B}$ and $\mathrm{C}$ of fig. 5 if the electron energy distribution is Maxwellian, which indeed applies, for we find for the SD a straight line over more than one decade. The distribution can be calculated from the SD curve following the formula given by Druyvesteyn $\left.{ }^{1}\right)$ :

$$
f(\mathrm{eV})=\frac{2}{e S}\left(\frac{2 \mathrm{mV}}{e}\right)^{\frac{1}{2}} \frac{\mathrm{d}^{2} i_{e}}{\mathrm{~d} V^{2}}
$$

where

$$
\begin{aligned}
& i_{e}=\text { the electron current to the probe } \\
& V=\text { probe potential with respect to space potential } \\
& e, m=\text { charge, mass of the electron } \\
& S \quad=\text { probe area }
\end{aligned}
$$

The electron energy distribution shown in fig. 5 (curve D) was calculated with the help of the measured $\mathrm{d}^{2} i / \mathrm{d} V^{2}$ curve, starting at $V=0$ multiplied by $V .5$ and normalized to one. The different temperatures found from the slope of curve $\mathrm{B}\left(5.58 \mathrm{~V}=64.800^{\circ} \mathrm{K}\right)$ from curve $\mathrm{C}\left(5.53 \mathrm{~V}=64.200^{\circ} \mathrm{K}\right)$ and from the maximum of curve $\mathrm{D}$

$$
V_{\max }-\frac{k T_{e}}{2 e}=2.8 \mathrm{~V} \rightarrow T_{e}=5.6 \mathrm{eV}=-65000^{\circ} \mathrm{K}
$$

are equal within $1 \%$.

IVd. Comparison of the three devices. We compared the three devices one after another, under the same tube conditions, at different pressures and different tube currents. Pressures of about 0.22 torr and 0.35 torr were used, and tube currents of 200,300 and $400 \mathrm{~mA}$.

In the course of the comparison we became aware of the fact that one has 
to be very careful about a low impedance in the SD circuit. For the devices 1 and 2 it was necessary to use resistances $R_{1}, R_{2}$ and $R_{3}$ (see fig. 1) of 5, 20 and $20 \Omega$ respectively, so that the modulation voltage was not influenced by the induced ac voltage generated between probe and earth. This perturbation voltage was mainly caused by the alternating probe current through the measuring resistance $R_{1}$. This voltage is in opposite phase with the modulation voltage, which results in a nonlinear decrease of the modulation voltage depending on the probe current. So it was necessary to choose $R_{1}$ as small as possible.

Furthermore it became clear that the tube current was influenced by the ac voltage put on the probe. That is to say, we measured an alternating current caused by the ac voltage on the probe. This voltage is proportional to the total impedance in the dc circuit. Consequently, the space potential near the probe varies with the same phase as the probe itself, so that the measured SD is lower especially in the maximum of the curve. We measured, for instance, in one case $50 \mathrm{mV}$ ac between anode and kathode, applying a modulation voltage of $0.8 \mathrm{~V}$ to the probe. This effect was found for all three frequencies. These difficulties were apparently due to the high impedance of the dc supply. They could be overcome by short circuiting the discharge tube with a suitable condensator, decreasing the impedance to the order of $10 \Omega$ or less.

After elimination of the above mentioned disturbances we compared with the utmost care the output of the three devices with respect to the shape of the SD curve and the zero cross. The measurements were performed with a modulation voltage of $0.8 \mathrm{~V}$. The shape of the curves was compared, normalizing the curves at $6 \mathrm{~V}$ negative with respect to space potential. Some typical results for different currents and pressures are presented in table III. The curves were taken from 0 to 40 volt (with respect to space potential); an enlarged curve was measured above 10 volt. The uncertainty in the measurement and the error in the evaluation of the measured curves is about 0.2 of the units used.

From the results presented and our other measurements it is no doubt clear that the devices 1 and 2 working at low frequencies, yield the same results within $1 \%$. A comparison with the results of device $3(200 \mathrm{kHz})$ gives a difference in the maximum of the SD curve of $10 \%$ to $25 \%$ depending on the pressure. This difference becomes less at higher voltages (see table III). The difference between the results obtained with the devices 1 and 2 and 3 is most probably due to the difference in the frequencies used. We believe that these differences are caused by the fact that at the high frequency $(200 \mathrm{kHz})$ the space charge sheath around the probe cannot adjust itself.

We have tried to go further into this difference in the SD curves by studying the dependence of the frequency. The whole measuring configuration of tube, probe and SD apparatus can for an alternating probe current, 
in the first order be replaced by an electrical circuit consisting of a resistor $R$ with parallel a capacitor $C$. The resistor replaces the quantity $\mathrm{d} V / \mathrm{d} I$ at the point of the current-voltage curve under consideration, while the capacitor $C$ replaces the stray capacities between probe and earth. So if an alternating voltage is put on the probe at any point of the probe curve at frequency $\omega$ it can be expected that the alternating probe current will increase with increasing $\omega$. We found, however, by putting on the probe a sinusoidal signal with a constant amplitude of $0.5 \mathrm{~V}$, from a variable oscillator $(5 \mathrm{kHz} \rightarrow$ $200 \mathrm{kHz}$ ), that the results were in contradiction with the above assumptions. We varied the frequency and measured the alternating probe current for different voltages on the probe. We found that the probe current increases about $10 \%$ with decreasing frequency in the range of $200 \mathrm{kHz} \rightarrow 40 \mathrm{kHz}$ in the neighbourhood of the space potential. This means that $R$ is a function of $\omega$, which is only possible if the sheath cannot follow the higher frequencies. Although these experiments do not give a complete solution of the problem, we believe that they also point to the conclusion that devices working with $40 \mathrm{kHz}$ or higher ${ }^{6}$ ) may give too low a value for the SD near the maximum of the curve.

If we compare our results, with respect to the temperature calculation from SD and from the electron energy distribution with the measurements of Vorob'eva ${ }^{6}$ ) et alii, we conclude that, in contradiction with their arguments, we are able to determine the space potential at the zero cross of the $\mathrm{SD}$ curve and do find from the slope of the SD and probe curve and from the maximum of the electron energy distribution $\left(V_{\max }=k T_{e} / 2 e\right)$ the same temperature within very narrow limits (see fig. 5). Vorob'eva et alii use an $\mathrm{SD}$ device of the third type $(80 \mathrm{kHz})$ which may cause difficulties just near the maximum of the SD curve, resulting in an error in the top of the distribution function.

Acknowledgements. The authors wish to thank Professor Dr J. A. Smit for his comments and helpful discussions of this paper. They also wish to express their gratitude to Mr G. J. Komen and Mr J. J. Langerak for the designing and building of the electronic equipment.

This work was performed as part of the research program of the association of Euratom and the "Stichting voor Fundamenteel Onderzoek der Materie" (F.O.M.) with financial support from the "Nederlandse Organisatie voor Zuiver Wetenschappelijk Onderzoek" (Z.W.O.) and Euratom. 


\section{REFERENCES}

1) Druyvesteyn, M. J., Z. Phys. 6\$ (1930) 781.

2) Sloane, H. R. and McGregor, E. I. R., Phil. Mag. 18 (1934) 193.

3) Boyd, R. L. F. and Twiddy, N. D., Proc. Roy. Soc. (London) $\mathbf{2 5 0}$ (1959) 53.

4) Malyshev, J. M. and Fedorov, V. L., Dokl. Akad. Nauk. U.S.S.R. 92 (1953) 269.

5) Branner, C. R., Friar, E. M. and Medicus, G., Rev, sci. Instrum. 44 (1963) 231.

6) Vorob'eva, N. A., Kagan, Yu. M, and Milenin, V. M., Sov. Phys.-Tech. Phys. 9 (1964) 632 and 8 (1963) 423.

7) Call, S. M., Rev. sci Instrum. 36 (1965) 850. 